\title{
Nurses' perspective of the research-practice gap in nursing
}

\author{
Clara Agbedia $^{1 *}$, Ijeoma Okoronkwo ${ }^{2}$, E. Onokayeigho ${ }^{1}$, Mary Ann Agbo ${ }^{2}$ \\ ${ }^{1}$ Department of Nursing Science, Delta State University, Abraka, Nigeria \\ ${ }^{2}$ Department of Nursing Science, University of Nigeria, Enugu Campus, Enugu State, Nigeria \\ Email: oniovo4life@yahoo.com
}

Received 3 December 2013; revised 4 January 2014; accepted 24 January 2014

Copyright (c) 2014 Clara Agbedia et al. This is an open access article distributed under the Creative Commons Attribution License, which permits unrestricted use, distribution, and reproduction in any medium, provided the original work is properly cited. In accordance of the Creative Commons Attribution License all Copyrights (C) 2014 are reserved for SCIRP and the owner of the intellectual property Clara Agbedia et al. All Copyright (C) 2014 are guarded by law and by SCIRP as a guardian.

\begin{abstract}
This work aims to explore the views of Nigerian nurses on research-practice gap in the clinical practice and the challenges in the utilization of nursing research. The results from in-depth interview and focus group discussion demonstrated that a researchpractice gap existed in the clinical setting. The nurses generally did not utilize research findings in their practice. Some useful suggestions were recommended to solve the problem.
\end{abstract}

\section{KEYWORDS}

\section{Nursing; Theory; Research; Practice; Research Utilization}

\section{INTRODUCTION}

Applying research findings in nursing practice is perhaps the greatest challenge facing the nursing profession today, though it is widely acknowledged that scientific research is central to the development of nursing and midwifery as professional disciplines [1]. However, there is a relative little evidence in the literature relating to the application of research findings into contemporary nursing practice especially in Nigeria. Nurse theorists over the years have identified potential benefits of nursing theory. This includes: articulation of what nursing is and what roles nurses play, offering ways of how nurses can help patients, a tool that renders practice more efficient and more effective, enhancing communication, increasing autonomy and accountability to care among others [2]. There are two possibilities for these. Perhaps this is because expectation of nursing theories and expectations of practicing nurses are unrealistic or perhaps nursing theories which

"Corresponding author. are useful for nursing practice have not yet been devised.

Until recently globally research in nursing was the prerogative and interest of nurse academician lecturing in nursing educational programs whereas nurse clinician think that they have nothing to contribute to nursing research. In Nigeria nursing care continues to be driven according to the procedure book rather than research driven, in spite of the increase in the quantity and quality of nursing research and the inclusion of research content in current nursing curricula by Nursing and Midwifery Council of Nigeria [3].

The purpose of this study was to explore the views of Nigerian nurses on research-practice gap in their clinical practice and the challenges faced in the utilization of nursing research. The essence is to remind Nigeria nurses on the role and the place of research in nursing in an embracing manner with the hope that nurses in different strata in the health care delivery system will appreciate the need to go back to the drawing board and come out with strategies on how to enforce research utilization in nursing practice. The premise is that nurses are increasingly expected to adopt a research-based practice which emphasizes decision based on the best available evidence and the use of outcome studies to guide practice.

Theory-Research-Practice Relationship Theory in nursing represents the individual and collective efforts of nurses to define and direct the profession and provides the basis for continued theoretical development [5]. On the basis of this theory, nurse scientists formulate hypotheses about what will occur in specific situations. These hypotheses are then subjected to empirical research. In order to be useful, two things are essential; first theory must arise from the systematic abstraction of nursing reality and the information acquired through nursing research must be developed into nursing theories that describe, explain or predict nursing phenomena. The 
theories must then be tested through further nursing research and then refined to enhance their usefulness in nursing practice.

Systematically developed theory will produce a wellfounded basis for the continued use of these purposes in nursing practice. It challenges thinking, provides new analytic skills, and helps all practitioners become more purposeful in their actions.

Theory-Research-Practice Relationship as discussed above could be argued that it is implicit in [6] accounts of the ways in which the theory-practice relationship in nursing is commonly understood. These are the "commonsense” approach, the "applied-science” approach, the "practical" approach and the "critical" approach. The "commonsense” approach suggests a practice driven theory, with theory being derived from practitioners' understanding and insights. Within the "applied-science" approach, the theory-practice relationship is one in which theory is viewed as abstract principles that may be brought to bear on practice in order to guide and regulate it. The "practical” approach represents a view, which upholds the art of deliberation and practical wisdom. The theory-practice relationship that is sustained by this approach is one in which theory informs practitioners' sense of what is good practice and, in this way, offers the practitioner a way of acting that is "right" and "just". This approach holds practice to be an ethical activity. The fourth way of viewing theory is the "critical” approach in which practitioners engage in critical self-reflection and, in so doing, can increase their self-knowledge and rational autonomy.

"Common-sense" approach is widely articulated within the literature. In this context theory originates in practice and is refined by research. To be complete, theory must return to practice in such a way that practice serve as the origin for ideas to guide practice. In reality, the "applied-science” approach is predominant in which nursing theory, practice, and research are hardly related at all. Nurse theorists are still developing theories in isolation, researchers pursue questions of interest only to educators and administrators and practitioners pursue their practice while oblivious to what the other two groups, researchers and educators were doing. This separation can be argued that it may have arisen because the type of knowledge which is valued by nurse theorists and nurse researchers are not seen as relevant by practicing nurses, who use a different type of knowledge in practice.

The linkage of findings in nursing education, research, and practice into a coherent structure as argued by [5] will make the body of accumulated nursing knowledge more accessible and useful.

\subsection{Research Questions}

The extent to which the research informs the practice of these nurses was explored by these questions.

- What is the relevance of research to practice?

- Do you use nursing research in your practice?

- What are the barriers in the use of research?

\subsection{Significance of the Study}

Nursing literature echoes the need for theory-based nursing $[4,5]$. The premise is that practice must be based on researches that are based on sound theory. Nurses need to move beyond non conventional nursing pattern to intervention using nursing knowledge that is holistic and specific to nursing concerns. This study highlights the challenges Nigeria nurses face in the use of nursing research, and how this problem can be solved to bring about the desired changes

\section{RESEARCH METHOD}

\subsection{Design}

A qualitative descriptive approach was used. This design provides a comprehensive summary of a phenomenon in everyday language and is ideal when direct descriptions of the phenomenon are desired.

\subsection{Sample}

A purposive sample of 60 registered nurses enrolled in a Bachelor of Nursing Science programme participated in the study. Participants were nurses with more than five years of nursing experience who were on in-service training who had worked full-time in four large hospitals covered by the Ministry of Health. These participants were selected from four different units in the four hospitals representing surgery, medicine, pediatrics and gynecological units at the time of the study. These units were selected because they are all non-intensive units and also to ensure maximum variations. Fifty-four participants were females and six were males. Ages ranged from 25 to 46 years. See Table 1 .

\subsection{Data Collection}

Data were collected using a semi structured interview guide and focus group discussion. Focus groups and interview provided a deep, rich understanding of these nurses perspective on topics [4]. Four focus groups discussions and three individual interviews were held with participants. Focus groups took an average of $30-60$ minute each. The interview was in two sessions, each session lasting approximately for 45 minutes. Data were collected and analyzed simultaneously, according to the grounded-theory approach. Each interview was transcribed verbatim and analyzed before the next interview took place. Therefore each interview provided the 
Table 1. Age distribution.

\begin{tabular}{ccc}
\hline Age & Frequency & Percentage (\%) \\
\hline $15-25$ & 19 & $32 \%$ \\
$26-35$ & 29 & $48 \%$ \\
$36-45$ & 10 & $17 \%$ \\
$46-55$ & 02 & $3 \%$ \\
Above 55 & - & - \\
Total & $\mathbf{6 0}$ & $\mathbf{1 0 0 \%}$ \\
\hline
\end{tabular}

direction for the next one.

\subsection{Data Analysis}

Data were collected and analyzed using a groundedtheory approach. The term grounded theory reflects the concept that theory emerging from this research is grounded in the data [5]. The grounded-theory approach has been used in nursing research since 1970 . Open, axial and selective coding was applied to the data [5]. Although the pparticipants were from different wards with varied qualification, themes that arose were consistent across interviews. Interviewing stopped when data saturation occurred. Data were considered "saturated" when no more code could be identified and the categories were "coherent". Thematic analysis was undertaken and it revealed several issues relating to 3 major themes; relevance of theory, clinical environment and barriers use of research Credibility of data was established through participants' revision and peer check to see whether the codes and themes were true to their experience. As a further validity check, two expert nurse researchers did peer checking on the transcripts. Results were also checked with some of the nurses who did not participate in the research to confirm the fitness of the results as well.

\subsection{Ethical Considerations}

The ethics committee of Faulty of Medical Sciences of the university gave permission for the study. Other ethical issues in this study involved the assurance of confidentiality and anonymity for the participants. All participants were informed of the purpose and design of the study and the voluntary nature of their participation. Written consent was sought from the participants.

\section{RESULTS}

\subsection{Demographic Data}

Three major themes and sub themes are;

- Relevance of theory to practice

- use of research in nursing
- Clinical environment

- workload/staff shortage

- role of the teacher

- Barriers use of research findings in nursing

\subsection{Relevance of Research to Practice}

One of the difficulties facing nursing researchers is the need to make it relevant to the practicing nurse. The entire participants agreed that nursing research is very useful in nursing practice.

Participant 1 said it gives me the assurance and the backing that what I am doing is right

Other reasons given include; helps to generate knowledge, help to decide what is known now and what we need to know, enhances the professional status of nursing

In assessing the use of research it is valuable to know their source of nursing knowledge.

From Table 2, 56\% of the nurses do not read journal. The implication of this is that these nurses are unlikely to utilize research findings in practice.

\subsection{Use of Research in Nursing}

It is now accepted that nursing research has reached a level of sophistication when it can be implemented and used for practice. In a much quoted article [7] gave reasons why nurses do not use research findings that are relevant to their practice. She suggested that it is because they do not hear about them. In an attempt to find out the extent at which these nurses read journals in this study, findings revealed that $44 \%$ of the nurses read journals while 56\% do not read journals. The argument being made here is that how can nurses in this study hear about research if they do not read journals. Although nursing research are disseminated through publication in books and nursing journals, the problem as noted by [8] is that much of it is not being read by nurses in general and particularly those in clinical practice. The reasons given by participants include: journals are not readily accessible, they are expensive and don't know how to utilize research findings in journal. The major reason for not reading journals (research findings in journal are not applicable in my practice 28\%) as seen in Table 3 below call for concern. In the word of one participant;

Examples seen in most American and UK studies are not relevant my nursing practice. Thus nurses must be

Table 2. Reading of journal.

\begin{tabular}{ccc}
\hline Read & Frequency & Percentage (\%) \\
\hline yes & 26 & 44 \\
No & 34 & 56 \\
Total & $\mathbf{6 0}$ & $\mathbf{1 0 0}$ \\
\hline
\end{tabular}


Table 3. Reasons for not reading journal.

\begin{tabular}{lcc}
\hline \multicolumn{1}{c}{ Option } & Frequency & Percentage (\%) \\
\hline Journals are not readily accessible & 8 & 13 \\
Journals are expensive & 12 & 20 \\
$\begin{array}{l}\text { Do not know how to utilize } \\
\text { esearch findings }\end{array}$ & 12 & 20 \\
$\begin{array}{l}\text { Research findings in journal } \\
\text { is not applicable in my practice }\end{array}$ & 28 & 47 \\
Total & $\mathbf{6 0}$ & $\mathbf{1 0 0}$ \\
\hline
\end{tabular}

encouraged to read the reports of published research in journals.

The response of participants on the extent at which they utilize research as shown in Table 4 shows that only (17\%) use research in practice while the majority (83\%) does not. The technical research language was the problem of another respondent. She explained that the language used is too technical to be understood by practice nurses. Participant 21 feels that nursing research does not take cognizance of the context of care. This opinion was collaborated when she pointed that some researches she has read in American and UK studies are not relevant to her practice, some pay less attention to the culture of the individual patient. To her this is essential ingredient in the nurse-patient relationship. Participant 30 in her words noted that "nursing research is too difficult and stressful to use in actual nursing practice, she went further, that the research is remote from everyday nursing”.

Therefore, constructing a research friendly culture through appropriate infrastructure is a way of promoting the use of research in practice. [9] reported a statistically significant relationship between research climate, an environment where research use is encouraged and recognized, and research utilization. Task-centered practice reinforces traditional based practice and this further segregates the nurses from research based practice. Rushing through responsibilities and conforming to superiors, as argued by often result in nurses caring for the wards more than the patients. Thus, nurse administrators, organizational managers and policymakers need to provide nurses with a supportive professional environment to speed research-based practice movement. The fact that nurses do as they are told in their daily practice without thinking about is captured in this participant view;

"The head nurse tells me what to do, whether to apply the dressing to a patient or give drugs". Therefore, I do not actively think for my caring for the individual patient [9].

\section{Use of Theories in Practice}

On the use of theories in practice as seen in Table 5 above reveals that, most of the respondents that apply theory in practice use Dorothy Orem (50\%) and Callister Roy (30\%). Other theories such as Imogen King, Hildegard Peplau were not common among the respondents.

In another comment, one participant asserted that... the available theories reflect other people's culture, not African man's culture and this affect its application in practice.

Participants' use of Dorothy Orem's theory may be attributed to the self care model which is commonly used in Nigeria. Furthermore, it incorporates the medical perspective. The non utilization of the other theories it can be argued is not unrelated to the fact that the concept of the environment is not well articulated. The neglect of the environment becomes a major problem in the utilization of the existing theories. Two theories (Nightingale \& Rogers) have addressed the influence of the environment on patient care.

\subsection{Clinical Environment}

Even though the clinical area is considered to be the centre of nursing, the ethos of the ward has a profound effect on the utilization of nursing research. In explaining the influence of workload, one respondent put it succinctly: "How can a nurse use her professional nursing knowledge effectively when you are alone in shift caring for 40 patients. She explained further that acute staff shortage mitigate the use of research in nursing."

Another participant reflecting on her experience as a nursing student felt that there is dichotomy between what is learnt in the classroom and what is practiced on the ward, thus resulting in the research-practice gap. She explained further-“in the classroom we were taught that the best method of patient care is team nursing but in the ward functional nursing is the order of the day. In this context how can you put into practice what you have learnt'?

Table 4. Utilization of research.

\begin{tabular}{ccc}
\hline Option & Frequency & Percentage (\%) \\
\hline Yes & 10 & 17 \\
No & 50 & 83 \\
Total & $\mathbf{6 0}$ & $\mathbf{1 0 0}$ \\
\hline
\end{tabular}

Table 5. Type of theory use in practice $(n=10)$.

\begin{tabular}{ccc}
\hline Theory & Frequency & Percentage \% \\
\hline Virginia Henderson & 2 & 20 \\
Dorothy Orem & 5 & 50 \\
Callister Roy & 3 & 30 \\
Total & $\mathbf{1 0}$ & $\mathbf{1 0 0}$ \\
\hline
\end{tabular}


Another participant related the culture-shock she experience as a nursing student she noted that the nursing knowledge given in the classroom cannot cater for the complex situations on the clinical wards.

One of the students summed up the research-practice gap dichotomy by saying 'nursing theory and research are for passing examination in the classroom and not applicable for actual practice'.

The views of the last two participants are in agreement with the views by [10] that many of the nursing theories were devised by nurse educators, who use theories as framework for teaching and require nursing students to use theoretical framework when they are learning how to assess patients and to plan care.

Other participants commented on the lack of direction and guidance on the ward. They argued that nursing students ideally are posted to the clinical area to put into practice what they have learnt but in reality they are often used to service needs. They explained further that on the ward, the patient expect you (student) to know all and when in doubt about some care issues and guidance trained staff they are too busy to be of assistance. According to them the way out is to have nurse educators on the ward with the students.

The central tenet here is that since learning occurs in two separate institutions, the classroom and the ward, the nurse educator should be the link between the two. This view is supported by [10]. He argued that since students are now supernumerary, nurse educators should spend quality time in the clinical area to ensure that students are able to link theory taught in classroom to practice on the ward.

\subsection{Barriers in the Use of Research}

The major barrier as reported by the participants is the implementation of research. One participant argued that organizational structure and clinical policy may not favour the application of research in practice. For example, in the use of evidence-based practice, the greatest barrier is effective use of information technology. This collaborate the finding of [3] that not enough computers was identified by the nurses (60\%) as being a barrier to computer use. Technical support for information technology systems was also limited

Another participant commented that research cannot be translated to practice as there are lots of improvisations due to inadequate material resources on the wards and the use of unskilled staff to carry out nursing activities. In the words of one participant" unskilled staff that is used to fill the gap of skilled staff does not have knowledge of recent nursing advances therefore procedural applications are faulty.

Many of the participants reiterated the inability of older nurses to direct the younger ones. This may be due largely to lack of knowledge on theory and its application. It was argued by [8] that four conditions have to be met if nurses are to implement research. These are; nurses must have the necessary knowledge, skill and attitudes, be able to overcome barriers to implementation, support for innovation must be available, innovation must be valued and rewarded. With respect to the first criterion, nurses in this study demonstrate positive attitudes towards research, but their access to and the use of research is limited. Therefore, adequate support for innovation is one important component of any strategy to improve the implementation of research.

When asked how may these barriers be removed? Participants proposed diverse ways.

- Nursing theories should be expressed in clear understandable terms.

- Researches should be applicable in real life situation.

- Nurse educators who teach these theories should extend such teachings to the clinical area if they want it to be put into practice.

\subsection{Implication}

\subsubsection{Developing Theory from Clinical Setting}

A model developed by [11] is relevant for bridging the gap between nursing research and practice. The strength of this model is that it assumes a problems solving approach that includes diagnosing a problem or need, analyzing or clarifying the problem, obtaining pertinent information, creating a considerable alternatives, developing and implementing action plan and finally evaluating and possibly refining the new knowledge skill or innovation. The resource system (nurse academicians, nurse theorists and nurse educators) and the user system (nurse clinician) must exchange ideas and views in a two-way interaction and continuously make the effort to stimulate each other's problem solving behavior. In this context it is envisaged that the resource system (nurse educators, academicians, researches) must appreciate the user's (nurse clinicians) internal needs and problem solving patterns. The user in turn must be able to appreciate the generation of new knowledge, skills and innovation relevant to her practice. Therefore this social interaction will not only make solution to nursing problems more relevant and effective but it will also build relationship of trust and mutual perception by the user and resource persons that the other is truly concerned. This is needed if nursing must be relevant in the age of high technology and medical advancement.

\subsubsection{New Pedagogies in Nursing Education}

Nurses' research utilization as part of evidence-based practice must be strongly emphasized in today's nursing 
education and clinical practice. The premise is that nurses are more likely to use research by the nature of their education and positive attitude to research. In nursing education, the most recent up to date research findings are available to students in the classroom. However, when these students enter the clinical nursing ward, the appropriate facilities to use the research information are not available. It is for this reason that it is difficult for nurses to carry over the information learned in school into the clinical nursing ward.

Nurse educators must turn to new pedagogies in educating nursing workforce. These new pedagogies must be research-based, responsive to the rapidly-changing health care system, and reflective of new partnerships between and among students, teachers and clinicians. The ultimate outcome of these efforts is evidence-based approaches to nursing education in which students learn to provide skillful and compassionate nursing care in fluid and uncertain health care environments.

\subsubsection{Clinical Mentorship in Research Process}

Clinical mentorship is also needed. Clinical mentorship is a system of practical training and consultation that fosters ongoing professional development to yield sustainable high-quality clinical care outcomes [12]. Within this context the purpose of this mentoring is to socialize clinical nurses to research process so that skills gained during education have effects on nursing practice. Clinical mentorship is therefore geared to increase nurses' ability to use research however; nurse educators must be flexible and be willing to mentor clinicians to promote research utilization. Ample opportunities exist during clinical mentoring to incorporate research based evidence in discussing issues relating to organization of care and treatment services, and reinforcing clinical decisions and diagnoses.

\subsubsection{Joint Appointments}

A major challenge to the nursing profession is to find ways of merging theory and practice in the delivery of nursing education and patient care. One option for achieving this goal is Joint appointments for nurse educators. Joint appointments refer to a variety of arrangements whereby concurrent employment occurs within an educational institution and a clinical setting. The major benefit of joint appointment of nurse educators as noted by $[13,14]$ is that it allows the nurse educators spend time in clinical practice updating their clinical skills and re-experiencing the realities of practice.

\section{CONCLUSION}

This presentation has highlighted the fact that nursing in Nigeria is still a long way from achieving research that influences practice. Nigerian nurses need to use sound research evidence in practice. Perhaps the best way to deal with this uncertainty of implementing research in practice is for nurse leaders to reassess the inherent factors that are influencing research utilization and come out with possible solution. Nursing administrators and educators must search for programs and strategies to establish research-based nursing practice.

\section{REFERENCES}

[1] Royal College of Nursing (2009) Research ethics: RCN guidance for nurses. RCN, London.

[2] Retsas A. (2000) Barriers to using research evidence in nursing practice. Journal of Advanced Nursing, 31, 599606. http://dx.doi.org/10.1046/j.1365-2648.2000.01315.x

[3] Agbedia, C.O. and Richards, F. (2013) Nurses use of information technology in their clinical practice in the Niger Delta Nigerian Research. Journal of Clinical Sciences, 2, 58-64.

[4] Polit, D.F. and Beck. C.T. (2002) Nursing research: Principles and methods. 6th Edition, J. B. Lippincott, Philadelphia.

[5] Diers, D. (2004) Research in nursing practice-Speaking nursing narratives of practice, research, policy \& other profession. Amazons publisher, Philadelphia

[6] Carr, W. (1986) Theories of theory and practice. Journal of Philosophy of Education, 21, 163-175.

[7] Hunt, J. (2000) Barriers to research utilization. Journal of Advanced Nursing, 23, 423-425. http://dx.doi.org/10.1111/j.1365-2648.1996.tb00001.x

[8] Jolley, S. (2002) Raising research awareness: A strategy for nurses. Nursing Standard, 16, 33-39. http://dx.doi.org/10.7748/ns2002.05.16.33.33.c3188

[9] Meijers, J., Janssen, M., Cummings, G., Wallin, L., Estabrooks, C. and Halfens, R. (2006) Assessing the relationships between contextual factors and research utilization in nursing: Systematic literature review. Journal of Advanced Nursing, 55, 622-635. http://dx.doi.org/10.1111/j.1365-2648.2006.03954.x

[10] Landers, M.G. (2000) The theory-practice gap in nursing Journal of advanced nursing. The Role of the Nurse Teacher, 32, 1550-1556.

[11] Havelock, R.G. (1972) Resource-user linkage and social problem solving. Center for Research on Utilization of Scientific Knowledge, Institute for Social Research, University of Michigan, Ann Arbor.

[12] Quinn, F.M. (2009) Principle and practice of nurse education. 4th Edition, Stanley Thornes Ltd., London.

[13] Williamson, G.R. and Webb, C. (2001) Supporting students in practice. Journal of Clinical Nursing, 2, 284-292. http://dx.doi.org/10.1046/j.1365-2702.2001.00478.x

[14] Noonan, B.J., Hughes, M., Hayes, C.C., Hartigan, I., O'Connell, L., Cummins, A. and Fehin, P. (2009) The effectiveness of the lecturer practitioner role in clinical practice: An Irish perspective. Nurse Education Today, 29, 561-565. http://dx.doi.org/10.1016/j.nedt.2008.12.007 